\title{
Between-cow variation in digestion and rumen fermentation variables associated with methane production
}

\author{
E. H. Cabezas-Garcia, ${ }^{\star 1}$ S. J. Krizsan, ${ }^{*}$ K. J. Shingfield, $\dagger^{2}$ and P. Huhtanen ${ }^{\star 1}$ \\ *Department of Agricultural Research for Northern Sweden, Swedish University of Agricultural Sciences, SE-901 83 Umeå, Sweden \\ †Institute of Biological, Environmental and Rural Sciences, Aberystwyth University, SY23 3EB Aberystwyth, United Kingdom
}

\begin{abstract}
A meta-analysis based on an individual-cow data set was conducted to investigate the effects of betweencow variation and related animal variables on predicted $\mathrm{CH}_{4}$ emissions from dairy cows. Data were taken from 40 change-over studies consisting of a total of 637 cow/period observations. Animal production and rumen fermentation characteristics were measured for 154 diets in 40 studies; diet digestibility was measured for 135 diets in 34 studies, and ruminal digestion kinetics was measured for 56 diets in 15 studies. The experimental diets were based on grass silage, with cereal grains or by-products as energy supplements, and soybean or canola meal as protein supplements. Average forage:concentrate ratio across all diets on a dry matter basis was 59:41. Methane production was predicted from apparently fermented substrate using stoichiometric principles. Data were analyzed by mixed-model regression using diet and period within experiment as random effects, thereby allowing the effect of experiment, diet, and period to be excluded. Dry matter intake and milk yield were more repeatable experimental measures than rumen fermentation, nutrient outflow, diet digestibility, or estimated $\mathrm{CH}_{4}$ yield. Between-cow coefficient of variation (CV) was 0.010 for stoichiometric $\mathrm{CH}_{4}$ per mol of volatile fatty acids and 0.067 for predicted $\mathrm{CH}_{4}$ yield $\left(\mathrm{CH}_{4} /\right.$ dry matter intake). Organic matter digestibility (OMD) also displayed little between-cow variation $(\mathrm{CV}=0.013)$, indicating that between-cow variation in diet digestibility and rumen fermentation pattern do not markedly contribute to between cow-variation in $\mathrm{CH}_{4}$ yield. Digesta passage rate was much more variable $(\mathrm{CV}=$ 0.08) between cows than OMD or rumen fermentation pattern. Increased digesta passage rate is associ-

\footnotetext{
Received October 26, 2016.

Accepted February 15, 2017.

${ }^{1}$ Corresponding authors: edward.cabezas.garcia@slu.se and pekka.

${ }^{2}$ Deceased September 11, 2016.
} huhtanen@slu.se
\end{abstract}

ated with improved energetic efficiency of microbial $\mathrm{N}$ synthesis, which partitions fermented substrate from volatile fatty acids and gases to microbial cells that are more reduced than fermented carbohydrates. Positive relationships were observed between $\mathrm{CH}_{4}$ per mol of volatile fatty acids versus OMD and rumen ammonia $\mathrm{N}$ concentration versus OMD; and negative relationships between the efficiency of microbial $\mathrm{N}$ synthesis versus OMD and digesta passage rate versus OMD, suggesting that the effects of these variables on $\mathrm{CH}_{4}$ yield were additive. It can be concluded that variations in OMD and efficiency in microbial $\mathrm{N}$ synthesis resulting from variations in digesta passage contribute more to between-animal variation in $\mathrm{CH}_{4}$ emissions than rumen fermentation pattern.

Key words: digestibility, rumen fermentation, passage rate, variance component

\section{INTRODUCTION}

Enteric $\mathrm{CH}_{4}$ emissions from ruminants represent a loss of dietary energy and contribute to greenhouse gas emissions. Depending on feeding level and diet composition, 2 to $12 \%$ of feed gross energy $(\mathbf{G E})$ can be lost as $\mathrm{CH}_{4}$ (Blaxter and Clapperton, 1965; Johnson and Johnson, 1995). Thus, strategies that mitigate $\mathrm{CH}_{4}$ emissions are not only environmentally beneficial, but can also result in greater efficiency of feed energy utilization by the animal. Methane production in cattle is strongly and positively correlated with DMI (e.g., Yan et al., 2000; Hristov et al., 2013). Because $\mathrm{CH}_{4}$ can be produced only from potentially digestible substrate, $\mathrm{CH}_{4}$ production in the rumen can be expected to be positively related to feed digestibility. In addition, diet composition, which is closely associated with digestibility, influences passage and digestion kinetics of feed particles in the gastrointestinal tract, and finally $\mathrm{CH}_{4}$ production.

Forage-to-concentrate ratio and dietary fat content are important variables influencing $\mathrm{CH}_{4}$ emissions per unit intake $\left(\mathrm{CH}_{4}\right.$ yield; Hristov et al., 2013; Ramin and Huhtanen, 2013). Low $\mathrm{CH}_{4}$ yields have been reported 
when high-concentrate feedlot diets are fed to growing cattle (Johnson and Johnson, 1995), reflecting increased propionate production from the higher supply of starch in these diets. Conversely, fat supplementation clearly decreases $\mathrm{CH}_{4}$ production (Moss et al., 2000). In addition to manipulation of diet composition, many other mitigation strategies (e.g., ionophores, electron acceptors, and plant bioactive compounds) have been extensively studied (Hristov et al., 2013).

The common assumption that $\mathrm{CH}_{4}$ production is affected mainly by the diet has been challenged since the large variation in $\mathrm{CH}_{4}$ emissions also has been attributed to animal factors (Ellis et al., 2007; Yan et al., 2009). In a study by Blaxter and Clapperton (1965), the coefficient of variation between-animal of $\mathrm{CH}_{4}$ yield was 7 to $8 \%$ in a respiration chamber study with sheep and cattle fed restrictively. In dairy cows fed ad libitum, the coefficient of variation for $\mathrm{CH}_{4}$ yield was considerably greater (8-18\%) when measured by the $\mathrm{SF}_{6}$ technique (Vlaming et al., 2008). Studies conducted in sheep have shown that the variation in ruminal digesta retention time or passage rate is related to $\mathrm{CH}_{4}$ emissions, with high $\mathrm{CH}_{4}$ emitters having a larger rumen volume and digesta pools than low emitters (Pinares-Patiño et al., 2003; Pinares-Patiño et al., 2011; Goopy et al., 2014). Other studies have shown that the host animal controls the archaea populations in the rumen (Weimer et al., 2010; Roehe et al., 2016). Although deep metagenomic and metatranscriptomic sequencing has shown similar abundance of methanogens and methanogenesis pathway genes in high and low $\mathrm{CH}_{4}$ emitters, the transcription of methanogenesis pathway genes was substantially increased in sheep with high $\mathrm{CH}_{4}$ yields (Shi et al., 2014). The mechanisms explaining the between-animal variation in $\mathrm{CH}_{4}$ emissions are not fully understood. The examination of the between-animal differences in a large data set originating from variations in digestion physiology and rumen microbial ecology could help to elucidate it.

Because the animal variation is likely to be under genetic control, one option to mitigate $\mathrm{CH}_{4}$ emissions that has been suggested is to select for animals that emit less. Pinares-Patiño et al. (2013) demonstrated that there is repeatable individual variation in this trait and part of this variation is genetic, but that the heritability estimate was lower for $\mathrm{CH}_{4}$ yield than for total daily $\mathrm{CH}_{4}$ emissions (0.13 and 0.29 , respectively). Therefore, in addition to heritability, further progress in genetic selection for low $\mathrm{CH}_{4}$ emitters also depends on better understanding of the variables involved in the observed between-animal variation of this trait. The objective of the present study was to evaluate between-cow variation and repeatability in digestion and fermentation variables contributing to $\mathrm{CH}_{4}$ emis- sions using a large data set from physiological digestion studies using a meta-analytical approach.

\section{MATERIALS AND METHODS}

\section{Data}

A meta-analysis was conducted to evaluate repeatability and between-animal variation in digestion and fermentation variables related to $\mathrm{CH}_{4}$ yield in dairy cows. The data were taken from studies on rumencannulated dairy cows, conducted using either a Latin square or switch-back design, in the Nordic countries: Finland (30 studies, 117 diets), Sweden (8 studies, 27 diets), and Norway (2 studies, 10 diets). The complete data set consisted of 637 cow/period observations, which were considered to be the experimental unit (Supplemental data file; https://doi.org/10.3168/ jds.2016-12206). A minimum pre-condition for inclusion of a study in the meta-analysis was that feed intake, BW, milk production data, and rumen fermentation variables were reported. In addition, diet digestibility in the total tract was determined in 34 studies, omasal flow of nutrients in 26 studies, and ruminal pool sizes and digestion kinetics in 15 studies.

The mean forage-to-concentrate ratio of the diets was 59:41 on a DM basis. The concentrate supplements consisted principally of cereal grains, fibrous by-products from the food industry, and protein supplements, typically canola and soybean meal. Grass silage was the main forage source, but in 9 studies it was partly replaced with legume or whole-crop cereal silage. The diets were fed ad libitum either as TMR 30 studies) or fixed amounts of concentrate with forage ad libitum (10 studies). In omasal flow studies, the intake was usually restricted to $95 \%$ of ad libitum intake to avoid variations in daily intake during digesta sampling. When some chemical components (starch and fat in concentrate ingredients) were not reported in a specific study (n $=6$ ), the missing values were taken from Finnish feed table values (LUKE, 2016). Production measurements included BW, feed intake, diet chemical composition, milk yield, and milk composition (fat, protein, lactose, and MUN). Energy-corrected milk was calculated according to Sjaunja et al. (1991).

Diet digestibility was determined by total feces collection (27 studies) or by fecal spot sampling (7 studies) using acid-insoluble ash (Van Keulen and Young, 1977) or indigestible neutral detergent fiber (iNDF; Huhtanen et al., 1994) as internal markers. Digesta flow measurements were conducted in 26 studies using the omasal sampling technique (Ahvenjärvi et al., 2000) with the triple-marker system (France and Siddons, 1986). Microbial $\mathrm{N}$ synthesis was determined using ${ }^{15} \mathrm{~N}$ 
as a microbial marker except for 2 studies where purine-based derivatives were used. Rumen pool size was determined by manual rumen evacuation and digestion and passage kinetic variables were calculated using the compartmental flux method (Ellis et al., 1994). The animals were fed twice daily. Rumen fluid samples were mostly collected over $12 \mathrm{~h}$ after morning feeding. The VFA ratios acetate/propionate and propionate/ butyrate were calculated, and the lipogenic:glucogenic ratio of VFA was determined as (acetate + butyrate)/ propionate. Production of both $\mathrm{CH}_{4}$ and $\mathrm{CO}_{2}$ per mole of VFA $\left(\mathbf{C H}_{4} \mathrm{VFA} ; \mathbf{C O}_{2} \mathbf{V F A}\right)$ was calculated based on VFA stoichiometry (Wolin, 1960):

$$
\begin{gathered}
\mathrm{CH}_{4} \mathrm{VFA}(\mathrm{mmol} / \mathrm{mol} \text { of VFA })= \\
0.5 \times \mathrm{C}_{2}-0.25 \times \mathrm{C}_{3}+0.5 \times \mathrm{C}_{4}, \\
\mathrm{CO}_{2} \mathrm{VFA}(\mathrm{mmol} / \mathrm{mol} \text { of VFA })= \\
0.5 \times \mathrm{C}_{2}+0.25 \times \mathrm{C}_{3}+1.5 \times \mathrm{C}_{4},
\end{gathered}
$$

where $\mathrm{C}_{2}, \mathrm{C}_{3}$, and $\mathrm{C}_{4}$ are molar proportions (mmol/ mol) of acetate, propionate, and butyrate, respectively, in the sum of these VFA.

Total $\mathrm{CH}_{4}$ was calculated from OM apparently digested in the rumen (OMADR) and rumen fermentation stoichiometry using the following equations:

OMADR (mol of hexose; $\left.\mathrm{C}_{6} \mathrm{~mol} / \mathrm{d}\right)=$

[OM intake $(\mathrm{kg} / \mathrm{d})$ - omasal OM flow $(\mathrm{kg} / \mathrm{d})] /$

$$
0.162(\mathrm{~kg} / \mathrm{mol})
$$

where the coefficient 0.162 is the molecular weight of hexose.

The VFA production per unit of hexose $\left(\mathrm{C}_{6}\right)$ fermented was calculated using stoichiometric principles as described by Van Soest (1994):

$$
\begin{gathered}
\operatorname{VFAC}_{6}(\mathrm{~mol} / \mathrm{mol})=6 /\left(2 \times \mathrm{C}_{2}+3 \times \mathrm{C}_{3}\right. \\
\left.+4 \times \mathrm{C}_{4}+\mathrm{CH}_{4} \mathrm{VFA}+\mathrm{CO}_{2} \mathrm{VFA}\right),
\end{gathered}
$$

where 6 is the number of $\mathrm{C}$ atoms in hexose; $\mathrm{C}_{2}, \mathrm{C}_{3}$, and $\mathrm{C}_{4}$ are molar proportions $(\mathrm{mol} / \mathrm{mol})$ of acetate, propionate, and butyrate, respectively; and $\mathrm{CH}_{4} \mathrm{VFA}$ and $\mathrm{CO}_{2} \mathrm{VFA}$ are $\mathrm{CH}_{4}$ and $\mathrm{CO}_{2}$ production per mole of VFA, respectively.

To account for the more reduced state of microbial cells (Hungate, 1966; Czerkawski, 1986; Van Soest, 1994), $\mathrm{CH}_{4}$ emissions were adjusted to a hydrogen ion $\left(\mathrm{H}^{+}\right)$uptake of $8.1 \mathrm{~g}$ per $\mathrm{kg}$ cells (Czerkawski, 1986). Microbial OM flow (MOM) at the omasum was calcu- lated assuming that $\mathrm{N}$ concentration in microbial $\mathrm{OM}$ was $80 \mathrm{~g} / \mathrm{kg}$. Methane production was also adjusted for biohydrogenation ( $\mathrm{BH}$; H-atoms) of fatty acids in the rumen, using an equation presented by Huhtanen et al. (2015b). This equation assumes a proportion of 0.95 for rate of lipolysis and 0.90 for rate of biohydrogenation. A factor of 1.8 for the average number of double bonds was considered and a molecular weight of $280 \mathrm{~g} / \mathrm{mol}$ was assumed for fatty acids. Total $\mathrm{CH}_{4}$ production was calculated as

$$
\begin{gathered}
\text { Total } \mathrm{CH}_{4}(\mathrm{~g} / \mathrm{d})=16 \times[(\mathrm{OMADR}(\mathrm{mol} \mathrm{C} 6 / \mathrm{d}) \\
\times \operatorname{VFAC}_{6}(\mathrm{~mol} / \mathrm{mol}) \times \mathrm{CH}_{4} \operatorname{VFA}(\mathrm{mol} / \mathrm{mol}) \\
-\operatorname{MOM}(\mathrm{kg} / \mathrm{d}) \times 8.1(\mathrm{~g} / \mathrm{kg})] /(4-\mathrm{BH} / 4),
\end{gathered}
$$

where OMADR, $\mathrm{VFAC}_{6}, \mathrm{CH}_{4} \mathrm{VFA}, \mathrm{MOM}$, and $\mathrm{BH}$ are as defined above, 16 is the molecular weight of $\mathrm{CH}_{4}$, and 4 is number of $\mathrm{H}$ atoms in $1 \mathrm{~mol} \mathrm{CH}_{4}$. No adjustments were made for $\mathrm{CH}_{4}$ production in the hindgut.

The values of $\mathrm{CH}_{4}$ production estimated from the amount of OMADR using stoichiometric principles were compared with values obtained using empirical equations from Yan et al. (2000) and Ramin and Huhtanen (2013). The Yan et al. (2000) equation predicts the proportion of $\mathrm{CH}_{4}$ energy in GE from feeding level and GE intake. The $\mathrm{CH}_{4}$ production by the Yan model was calculated as follows:

$$
\begin{gathered}
\mathrm{CH}_{4}-\operatorname{Yan}(\mathrm{g} / \mathrm{d})=1,000(\mathrm{~g} / \mathrm{kg}) \times[-0.0078 \\
\times(\mathrm{FL}-1)+0.0877)] \times \mathrm{DMI}(\mathrm{kg} / \mathrm{d}) \\
\times 18.5(\mathrm{MJ} / \mathrm{kg} \mathrm{DM}) / 55.5(\mathrm{MJ} / \mathrm{kg}),
\end{gathered}
$$

where FL is feeding level multiple of maintenance, 18.5 is assumed GE concentration (MJ/ $\mathrm{kg}$ of $\mathrm{DM})$, and 55.5 is $\mathrm{GE}$ of $\mathrm{CH}_{4}(\mathrm{MJ} / \mathrm{kg})$.

The original equation proposed by Ramin and Huhtanen (2013, Equation [17]) was expressed in liters per day by multiplying the model coefficients by $\mathrm{CH}_{4}$ molecular weight of $16 \mathrm{~g} / \mathrm{mol}$ and dividing it by $22.4 \mathrm{~L} /$ mol (conversion factor), as follows:

$$
\begin{gathered}
\mathrm{CH}_{4}-\mathrm{RH}(\mathrm{g} / \mathrm{d})=1,000(\mathrm{~g} / \mathrm{kg}) \\
\times\left(3.6-0.25 \times \mathrm{DMIBW}+0.022 \times \mathrm{OMD}_{\mathrm{m}}-0.031\right. \\
\times \mathrm{EE}+0.013 \times \mathrm{NDF}+0.013 \times \mathrm{NFC}),
\end{gathered}
$$

where DMIBW is DMI/BW $(\mathrm{g} / \mathrm{kg}), \mathrm{OMD}_{\mathrm{m}}$ is $\mathrm{OM}$ digestibility determined at the maintenance level of feeding $(\mathrm{g} / \mathrm{kg})$, and dietary concentrations $(\mathrm{g} / \mathrm{kg}$ of $\mathrm{DM})$ of ether extract (EE), NDF, and NFC. 


\section{Statistical Analysis}

The PROC MIXED in SAS (version 9.3, SAS Institute Inc., Cary, NC) was used to calculate the variance components of the selected variables taking into account as random factors experiment (Exp), diet within experiment [Diet(Exp)], period within experiment $[\operatorname{Period}(\operatorname{Exp})]$, and cow within experiment [Cow(Exp)]. Covariance structure was defined in the model using the TYPE $=\mathrm{VC}$ (variance components) option in the RANDOM statement. The standard deviation and coefficient of variation for each factor were calculated as the square root of the variance estimate and standard deviation divided by the respective mean value of each factor.

Repeatability values (Rep) for the most relevant variables associated with enteric methane production were calculated as

$$
\operatorname{Rep}=\sigma_{\text {Cow }}^{2} /\left(\sigma_{\text {Cow }}^{2}+\sigma_{\text {Residual }}^{2}\right)
$$

where $\sigma_{\text {Cow }}^{2}$ and $\sigma_{\text {Residual }}^{2}$ are $\operatorname{Cow}(\operatorname{Exp})$ and residual variances, respectively. Repeatability values provide an estimate of the correlation between values from consecutive samples on the same cow, on the same diet, and within the same period of the same experiment.

Empirical models were developed between the variables of interest regarding their biological value by single regression analysis within the MIXED procedure of SAS (Littell et al., 2006), using the following model:

$$
Y_{i j}=B_{0}+B_{1} X_{1 i j}+b_{0}+b_{1} X_{1 i j}+e_{i j}
$$

where $Y_{i j}=$ the expected value for the dependent variable $Y$ observed at level of $j$ of the independent variable $X$ in study $i ; B_{0}=$ the overall intercept (fixed effect); $b_{0}=$ the random effect of study $i$ on the intercept $(i=$ $1, \ldots, 40) ; B_{1}$ is the regression coefficient of $Y$ on $X_{1}$ of $Y$ across all studies (fixed effects), $X_{1 i j}=$ value $j$ of the continuous variable $X_{1}$ in study $i ; b_{i}=$ is the random effect of study $i$ on the regression coefficient of $Y$ on $X_{1}$ in study $i(i=1, \ldots, 40)$, and $e_{i j}=$ the residual error.

The models included 2 random statements: a random intercept and slope of $X_{1}$ with SUBJECT = Diet(Exp), and a random intercept with SUBJECT = Period(Exp), using the TYPE $=\mathrm{VC}$ as the covariance structure for both random statements. The method $=$ ML (maximum likelihood) statement was used in the PROC MIXED model syntax. Only one random independent variable was used to avoid overparameterized models and improve convergence (St-Pierre, 2001).

Outlier observations for predicted $\mathrm{CH}_{4}$ production were investigated for leverage and influence by the

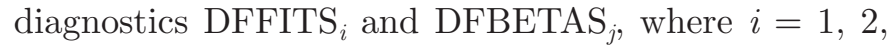

$\ldots, 637$ and $j, i$ denote the $j$ th regression coefficient in the regression equation $(=0$ or 1$)$ estimated without observation $i$, where $i=1,2, \ldots, 637$, respectively (Belsley et al., 1980). Cut-off values suggesting that an observation warranted examination were set at $\mid$ DFFITS $_{i} \mid>2 \sqrt{ }(p / n)$ and $\mid$ DFBETAS $_{i j} \mid>2 \sqrt{ } n$, where $p=$ number of variables estimated in the model and $n=$ total number of observations in the model. Dry matter intake and OMD were used as independent variables in the model when examining outliers. In total, 18 observations for predicting $\mathrm{CH}_{4}$ emissions (Table 3) were deleted from the data set because they were identified as outliers in the model as mentioned above.

\section{RESULTS}

\section{Data Description}

Mean and ranges of diet composition and animal production data are shown in Table 1, whereas rumen fermentation, diet digestibility, and digestion kinetics parameters are presented in Table 2. Despite the large differences in diet composition, rumen $\mathrm{pH}$ and the proportions of major VFA did not vary greatly compared with the proportions of minor VFA. The large variation in rumen ammonia $\mathrm{N}$ (RAN) was related to differences in feed sources, especially dietary $\mathrm{CP}$ concentration. The mean diet digestibility was high (OMD $740 \mathrm{~g} / \mathrm{kg}$ ), especially NDF digestibility (NDFD), as expected for dairy cows diets based mainly on good quality grass silage $(\mathrm{CV}=0.119$; Table 2$)$. The data set did not include any low digestibility diets. Digestion rate of potentially digestible NDF (pdNDF) displayed large variation in forage fiber composition among diets, ranging from 2.0 to $14.0 \% / \mathrm{h}$.

The values for predicted $\mathrm{CH}_{4}$ production are presented in Table 3. The $\mathrm{CH}_{4} \mathrm{VFA}$ exhibited low variation (CV $=0.043)$ considering that it also included between-diet and between-period variation. The variation was much greater $(\mathrm{CV}=0.28)$ for predicted total $\mathrm{CH}_{4}$ production $(\mathrm{g} / \mathrm{d})$, double that of the $\mathrm{CH}_{4}$ values predicted by empirical equations. The $\mathrm{CH}_{4}$ yield ranged from 8.9 to $37 \mathrm{~g} / \mathrm{kg}$ of DMI.

\section{Variance Components}

Dry matter intake has often influenced measures of ruminal digestion and $\mathrm{CH}_{4}$ production, but in the present study did not exert any influence on the variance component Cow(Exp) when used as a covariate in the statistical analysis, and was therefore omitted. In general, variation associated with the effect of experiment (Exp) was the largest source of variation observed in the data set (not shown). In Tables 4 to 8 , the variance 
Table 1. Description of diet composition, feed intake, and milk yield in the data set, which was derived from 40 experiments in dairy cows

\begin{tabular}{|c|c|c|c|c|c|}
\hline Item & $\mathrm{n}$ & Mean & $\mathrm{SD}$ & Minimum & Maximum \\
\hline \multicolumn{6}{|l|}{ Intake, kg/d } \\
\hline Forage DM & 637 & 11.1 & 2.68 & 2.5 & 18.9 \\
\hline Concentrate DM & 637 & 7.7 & 2.63 & 0.0 & 17.3 \\
\hline Total DM & 637 & 18.9 & 3.35 & 7.4 & 27.5 \\
\hline \multicolumn{6}{|c|}{ Diet composition, $\mathrm{g} / \mathrm{kg}$ of $\mathrm{DM}$} \\
\hline $\mathrm{OM}$ & 637 & 924 & 39.7 & 885 & 967 \\
\hline $\mathrm{CP}$ & 637 & 160 & 21.3 & 121 & 250 \\
\hline NDF & 637 & 394 & 54.8 & 246 & 549 \\
\hline Starch & 605 & 152 & 64.6 & 0.0 & 400 \\
\hline $\mathrm{NFC}^{1}$ & 589 & 327 & 62.2 & 109 & 506 \\
\hline Ether extract & 589 & 42.5 & 14.02 & 14.0 & 106 \\
\hline Fatty acids & 589 & 29.7 & 12.69 & 13.4 & 84.8 \\
\hline \multicolumn{6}{|c|}{ Milk yield and composition } \\
\hline Milk, $\mathrm{kg} / \mathrm{d}$ & 621 & 26.6 & 6.86 & 5.0 & 44.6 \\
\hline $\mathrm{ECM}, \mathrm{kg} / \mathrm{d}$ & 621 & 26.8 & 6.14 & 5.2 & 47.4 \\
\hline Fat, $\mathrm{g} / \mathrm{kg}$ & 621 & 41.2 & 6.05 & 16.5 & 56.9 \\
\hline Protein, $\mathrm{g} / \mathrm{kg}$ & 621 & 33.9 & 4.47 & 21.3 & 58.0 \\
\hline Lactose, $\mathrm{g} / \mathrm{kg}$ & 621 & 47.1 & 3.46 & 28.9 & 53.7 \\
\hline MUN, mg/dL & 435 & 11.0 & 3.93 & 3.3 & 27.0 \\
\hline \multicolumn{6}{|l|}{ Feed efficiency } \\
\hline $\mathrm{ECM} / \mathrm{DMI}, \mathrm{kg} / \mathrm{kg}$ & 621 & 1.39 & 0.235 & 0.40 & 2.02 \\
\hline $\mathrm{MNE}^{2}{ }^{2} \mathrm{~g} / \mathrm{kg}$ & 621 & 292 & 60.5 & 95.0 & 555 \\
\hline $\mathrm{BW}, \mathrm{kg}$ & 534 & 619 & 67.0 & 457 & 808 \\
\hline
\end{tabular}

${ }^{1} \mathrm{NFC}=1,000-$ ash $-\mathrm{CP}-$ ether extract $-\mathrm{NDF}$.

${ }^{2} \mathrm{MNE}=$ efficiency of $\mathrm{N}$ utilization for milk production, defined as milk $\mathrm{N} / \mathrm{N}$ intake.

components for Diet(Exp), Cow(Exp), and Residual are presented, to separate dietary and individual animal effects.

Variance component estimates for digestion variables are presented in Table 4. The variance component $\operatorname{Diet}(\operatorname{Exp})$ was the greatest source of variation. For NDFD, the variation due to Diet(Exp) was higher than that due to $\operatorname{Cow}(\operatorname{Exp})(\mathrm{CV}=0.057$ and 0.023 , respectively). Repeatability of NDFD was lower (0.26) than for OMD (0.37). The observed Cow(Exp) variation in $\mathrm{OMD}$ in the data set was rather small $(\mathrm{CV}=0.013)$. The proportion of rumen digestion in total NDF digestion varied more than the total NDF, and it was also a more repeatable measurement than total NDFD (0.34). Although highly significant and moderately repeatable (0.37) quantitatively, Cow (Exp) variation in metabolic fecal OM output (fecal OM - fecal NDF; g/ $\mathrm{kg}$ of DMI) was small $(\mathrm{SD}=3.9 \mathrm{~g} / \mathrm{kg})$.

The variance components for rumen fermentation patterns are shown in Table 5. Both rumen $\mathrm{pH}$ and total VFA were considerably more repeatable (Rep = 0.46) than molar proportions of individual VFA. For the molar proportions of acetate and propionate, the variation associated with diet was about double the between-cow variation, whereas for butyrate the diet and between-cow variance components were similar.

Overall, the between-cow variation in rumen fermentation pattern $\left(\mathrm{CH}_{4} \mathrm{VFA}\right)$ was only 0.010 , and repeatability was rather low (0.11). For the total $\mathrm{CH}_{4}$ production predicted from fermentation stoichiometry, variations associated with $\operatorname{Diet}(\operatorname{Exp})$ and $\operatorname{Cow}(\operatorname{Exp})$ were both greater in $\mathrm{CH}_{4}$ production predicted by empirical models (Table 6). Methane yield was less variable, both between diets and between cows, than total $\mathrm{CH}_{4}$ production. It is worth noting that $\operatorname{Diet}(\operatorname{Exp})$ variation was larger than $\mathrm{Cow}(\mathrm{Exp})$ only for $\mathrm{CH}_{4} \mathrm{VFA}$, and not the other approaches for predicting enteric $\mathrm{CH}_{4}$ production.

Estimates of variance components for $\mathrm{N}$ metabolism variables are presented in Table 7 . The Cow (Exp) variance component for RAN was of greater magnitude than the other $\mathrm{N}$ metabolism variables $(\mathrm{CV}=0.149)$. The repeatability of RAN was similar to that of efficiency of microbial N synthesis per kg of OMTDR (Rep $=0.35$ and 0.34 , respectively). Microbial $\mathrm{N}$ flow showed the highest repeatability value among $\mathrm{N}$ metabolism variables $(\operatorname{Rep}=0.51)$. The extent of between-cow variation in RAN also was larger than for other $\mathrm{N}$ metabolism variables $(\mathrm{CV}=0.149)$. In both the $\mathrm{OM}$ and NDF pools per kilogram of BW, the contribution of the Cow(Exp) variance component to the observed variation was larger than that of the Diet(Exp) variance component (Table 8).

\section{Empirical Models-Univariate Regressions}

Stoichiometric $\mathrm{CH}_{4} \mathrm{VFA}$ decreased $(P<0.01)$ with increasing DMI (Table 9), but it was not significantly 
Table 2. Description of rumen fermentation, digestibility, and $\mathrm{N}$ flow at the omasum in the data set, which was derived from 40 experiments in dairy cows

\begin{tabular}{|c|c|c|c|c|c|}
\hline Item $^{1}$ & $\mathrm{n}$ & Mean & $\mathrm{SD}$ & Minimum & Maximum \\
\hline \multicolumn{6}{|l|}{ Rumen fermentation } \\
\hline Rumen pH & 597 & 6.36 & 0.281 & 5.70 & 7.22 \\
\hline Total VFA, mmol/L & 636 & 113 & 17.0 & 71.0 & 162 \\
\hline \multicolumn{6}{|l|}{ Molar proportion, $\mathrm{mmol} / \mathrm{mol}$} \\
\hline Acetate $(\mathrm{A})$ & 636 & 654 & 25.3 & 547 & 729 \\
\hline Propionate $(\mathrm{P})$ & 636 & 181 & 19.7 & 123 & 287 \\
\hline Butyrate (B) & 636 & 125 & 18.7 & 75.2 & 195 \\
\hline Isobutyrate & 636 & 8.3 & 1.62 & 4.4 & 15.0 \\
\hline Valerate & 571 & 14.7 & 3.87 & 4.1 & 35.8 \\
\hline Isovalerate & 563 & 13.2 & 4.61 & 5.4 & 37.3 \\
\hline \multicolumn{6}{|l|}{ VFA ratios } \\
\hline $\mathrm{A} / \mathrm{P}$ & 636 & 3.66 & 0.479 & 2.05 & 5.71 \\
\hline$(\mathrm{A}+\mathrm{B}) / \mathrm{P}$ & 636 & 4.36 & 0.555 & 2.33 & 6.79 \\
\hline $\mathrm{RAN}, \mathrm{mg} / \mathrm{dL}$ & 617 & 9.34 & 4.804 & 1.50 & 34.0 \\
\hline \multicolumn{6}{|l|}{ Total-tract digestibility, $\mathrm{g} / \mathrm{kg}$} \\
\hline $\mathrm{OM}$ & 558 & 740 & 39.9 & 627 & 892 \\
\hline $\mathrm{CP}$ & 558 & 684 & 51.4 & 519 & 876 \\
\hline NDF & 542 & 648 & 77.2 & 413 & 864 \\
\hline MFOM, $\mathrm{g} / \mathrm{kg}$ & 542 & 100 & 20.0 & 54 & 172 \\
\hline \multicolumn{6}{|l|}{ Flow at the omasum } \\
\hline $\mathrm{OM}, \mathrm{kg} / \mathrm{d}$ & 397 & 8.21 & 2.14 & 2.10 & 14.0 \\
\hline $\mathrm{NDF}, \mathrm{kg} / \mathrm{d}$ & 405 & 2.88 & 0.986 & 0.385 & 5.71 \\
\hline NAN, $g / d$ & 371 & 433 & 119.5 & 113 & 732 \\
\hline Microbial N, g/d & 404 & 290 & 84.0 & 71 & 496 \\
\hline \multicolumn{6}{|l|}{ Microbial N efficiency } \\
\hline Microbial N, $\mathrm{g} / \mathrm{kg}$ of DMI & 404 & 15.7 & 2.90 & 8.4 & 23.2 \\
\hline Microbial N, g/kg of OMTDR & 380 & 23.6 & 5.24 & 10.7 & 50.7 \\
\hline \multicolumn{6}{|l|}{ Rumen pool size, $\mathrm{g} / \mathrm{kg}$ of $\mathrm{BW}$} \\
\hline DM & 217 & 18.4 & 3.70 & 9.6 & 30.5 \\
\hline $\mathrm{OM}$ & 217 & 16.6 & 3.47 & 8.5 & 29.0 \\
\hline $\mathrm{NDF}$ & 217 & 10.1 & 2.59 & 3.3 & 18.6 \\
\hline RNDFD, $\mathrm{g} / \mathrm{kg}$ & 404 & 608 & 105.3 & 245 & 878 \\
\hline \multicolumn{6}{|l|}{ Ruminal digestion kinetics, $\% / \mathrm{h}$} \\
\hline pdNDF-kd & 154 & 4.8 & 1.96 & 1.92 & 14 \\
\hline pdNDF-kp & 154 & 1.3 & 0.34 & 0.53 & 2.2 \\
\hline iNDF-kp & 154 & 2.6 & 0.62 & 1.34 & 4.6 \\
\hline
\end{tabular}

${ }^{1} \mathrm{RAN}=$ rumen ammonia $\mathrm{N} ; \mathrm{MFOM}=$ metabolic fecal OM; OMTDR $=$ OM truly digested in the rumen; RNDFD = rumen NDF digestibility; pdNDF-kd = digestion rate of potentially digestible NDF (pdNDF); pdNDF-kp = passage rate of pdNDF; iNDF-kp = passage rate of indigestible NDF.

Table 3. Description of predicted $\mathrm{CH}_{4}$ production for the data set, which was derived from 40 experiments in dairy cows

\begin{tabular}{lccccc}
\hline Item & $\mathrm{n}$ & Mean & $\mathrm{SD}$ & Minimum & Maximum \\
\hline Stoichiometric balance & & & & & \\
$\mathrm{CH}_{4}$ VFA, mmol/mol & 636 & 358 & 15.4 & 275 & 404 \\
$\mathrm{CO}_{2}$ VFA, mmol/mol & 636 & 583 & 21.0 & 508 & 659 \\
$\mathrm{CH}_{4}$ per mol of hexose & 636 & 634 & 27.6 & 507 & 718 \\
$\mathrm{Methane} \mathrm{emissions}_{\text {Total } \mathrm{CH}_{4},{ }^{2} \text { g/d }}$ & 360 & 403 & 111.9 & 142 & 674 \\
$\mathrm{CH}_{4} \mathrm{yield}^{3} \mathrm{~g} / \mathrm{kg}$ of DMI & 360 & 22.6 & 5.16 & 8.9 & 37.2 \\
$\mathrm{CH}_{4}-\mathrm{Yan},{ }^{4} \mathrm{~g} / \mathrm{d}$ & 360 & 391 & 58.6 & 197 & 509 \\
$\mathrm{CH}_{4}-\mathrm{RH},{ }^{5} \mathrm{~g} / \mathrm{d}$ & 360 & 381 & 61.0 & 192 & 539 \\
\hline
\end{tabular}

${ }^{1}$ Predicted from VFA stoichiometry (Equations [1] and [2]).

${ }^{2}$ Total $\mathrm{CH}_{4}$ was estimated from the amount of $\mathrm{OM}$ apparently digested in the rumen adjusted by $\mathrm{H}^{+}$sinks (Equation [5]).

${ }^{3}$ Calculated as total $\mathrm{CH}_{4} / \mathrm{DMI}$.

${ }^{4}$ Empirical model proposed by Yan et al. (2000; Equation [6]).

${ }^{5}$ Empirical model proposed by Ramin and Huhtanen (2013; Equation [7]). 
Table 4. Variance component estimates of digestion variables, developed using the data set derived from 40 crossover studies in dairy cows

\begin{tabular}{|c|c|c|c|c|c|}
\hline Item $^{1}$ & Estimate & $\mathrm{SE}$ & $P>\mathrm{Z}^{2}$ & $\mathrm{SD}^{3}$ & $\mathrm{CV}^{4}$ \\
\hline \multicolumn{6}{|c|}{$\mathrm{OMD}, \mathrm{g} / \mathrm{kg}, \mathrm{n}=558$} \\
\hline $\operatorname{Diet}(\operatorname{Exp})$ & 365 & 58.6 & $<0.001$ & 19.1 & 0.026 \\
\hline Cow (Exp) & 98 & 20.4 & $<0.001$ & 9.9 & 0.013 \\
\hline Residual & 170 & 16.6 & $<0.001$ & 13.0 & 0.018 \\
\hline \multicolumn{6}{|c|}{ NDFD, $\mathrm{g} / \mathrm{kg}, \mathrm{n}=542$} \\
\hline $\operatorname{Diet}(\operatorname{Exp})$ & 1,340 & 215.0 & $<0.001$ & 36.6 & 0.057 \\
\hline Cow(Exp) & 212 & 54.9 & $<0.001$ & 14.6 & 0.023 \\
\hline Residual & 596 & 59.2 & $<0.001$ & 24.4 & 0.038 \\
\hline \multicolumn{6}{|c|}{$\mathrm{RNDFD}, \mathrm{g} / \mathrm{kg}, \mathrm{n}=404$} \\
\hline $\operatorname{Diet}(\operatorname{Exp})$ & 2,324 & 446.9 & $<0.001$ & 48.2 & 0.079 \\
\hline Cow (Exp) & 740 & 186.9 & $<0.001$ & 27.2 & 0.045 \\
\hline Residual & 1,441 & 167.7 & $<0.001$ & 38.0 & 0.062 \\
\hline \multicolumn{6}{|c|}{ MFOM, $\mathrm{g} / \mathrm{kg}, \mathrm{n}=542$} \\
\hline $\operatorname{Diet}(\operatorname{Exp})$ & 43.9 & 8.23 & $<0.001$ & 6.62 & 0.066 \\
\hline $\operatorname{Cow}(\operatorname{Exp})$ & 14.9 & 4.03 & $<0.001$ & 3.87 & 0.039 \\
\hline Residual & 44.5 & 4.45 & $<0.001$ & 6.67 & 0.066 \\
\hline
\end{tabular}

${ }^{1} \mathrm{OMD}=$ total-tract $\mathrm{OM}$ digestibility; NDFD = total-tract NDF digestibility; RNDFD = proportion of ruminal digestibility of total NDF digestibility; MFOM = metabolic fecal OM. Exp = experiment; Diet $(\operatorname{Exp})=$ diet within experiment; $\operatorname{Cow}(\operatorname{Exp})=$ cow within experiment.

${ }^{2}$ Probability of Z-value.

${ }^{3}$ Calculated as the square root of the variance component estimate.

${ }^{4}$ Calculated as SD divided by the respective mean value of the variable.

related to rumen $\mathrm{pH}$. It was positively related to digestibility variables, RAN, and molar proportion of branched-chain VFA (BCVFA). For each kilogram increase in DMI, $\mathrm{CH}_{4} \mathrm{VFA}$ decreased by $0.95 \mathrm{mmol} / \mathrm{mol}$.

Digestibility of $\mathrm{OM}$ was negatively associated $(P$ $<0.01)$ with DMI, and the variation in OMD was closely related to NDFD and the proportion of ruminal digestion in total NDFD (Table 10). For the rumen evacuation data set, OMD was positively related to the digestion rate and negatively to the passage rate of pdNDF. For individual VFA, the molar proportions of acetate and butyrate were positively and the molar

Table 5. Variance component estimates of rumen fermentation variables, developed using the data set derived from 40 crossover studies in dairy cows

\begin{tabular}{|c|c|c|c|c|c|}
\hline Item $^{1}$ & Estimate & SE & $P>\mathrm{Z}^{2}$ & $\mathrm{SD}^{3}$ & $\mathrm{CV}^{4}$ \\
\hline \multicolumn{6}{|c|}{ Rumen $\mathrm{pH}, \mathrm{n}=597$} \\
\hline $\operatorname{Diet}(\operatorname{Exp})$ & 0.006 & 0.0016 & $<0.001$ & 0.0790 & 0.012 \\
\hline Cow(Exp) & 0.018 & 0.0031 & $<0.001$ & 0.1341 & 0.021 \\
\hline Residual & 0.021 & 0.0019 & $<0.001$ & 0.1437 & 0.023 \\
\hline \multicolumn{6}{|c|}{ Total VFA, $\mathrm{mmol} / \mathrm{L}, \mathrm{n}=636$} \\
\hline $\operatorname{Diet}(\operatorname{Exp})$ & 12.2 & 3.00 & $<0.001$ & 3.50 & 0.031 \\
\hline Cow (Exp) & 31.7 & 5.41 & $<0.001$ & 5.63 & 0.050 \\
\hline Residual & 37.5 & 3.42 & $<0.001$ & 6.13 & 0.054 \\
\hline \multicolumn{6}{|c|}{ Molar proportions of VFA, $\mathrm{mmol} / \mathrm{mol}$} \\
\hline \multicolumn{6}{|c|}{ Acetate, $\mathrm{n}=636$} \\
\hline $\operatorname{Diet}(\operatorname{Exp})$ & 222 & 34.6 & $<0.001$ & 14.9 & 0.022 \\
\hline Cow (Exp) & 55 & 11.9 & $<0.001$ & 7.4 & 0.011 \\
\hline Residual & 138 & 12.4 & $<0.001$ & 11.8 & 0.017 \\
\hline \multicolumn{6}{|c|}{ Propionate, $\mathrm{n}=636$} \\
\hline $\operatorname{Diet}(\operatorname{Exp})$ & 108 & 21.1 & $<0.001$ & 10.4 & 0.055 \\
\hline Cow(Exp) & 21 & 9.8 & 0.014 & 4.64 & 0.025 \\
\hline Residual & 185 & 16.3 & $<0.001$ & 13.6 & 0.072 \\
\hline \multicolumn{6}{|c|}{ Butyrate, $\mathrm{n}=636$} \\
\hline Diet(Exp) & 62.6 & 11.8 & $<0.001$ & 7.91 & 0.061 \\
\hline Cow(Exp) & 43.4 & 9.38 & $<0.001$ & 6.59 & 0.051 \\
\hline Residual & 96.1 & 8.71 & $<0.001$ & 9.80 & 0.075 \\
\hline
\end{tabular}

${ }^{1} \operatorname{Exp}=$ experiment; Diet $(\operatorname{Exp})=$ diet within experiment; Cow $(\operatorname{Exp})=$ cow within experiment.

${ }^{2}$ Probability of Z-value.

${ }^{3}$ Calculated as the square root of the variance-component estimate.

${ }^{4}$ Calculated as SD divided by the respective mean value of the variable. 
Table 6. Variance component estimates for enteric $\mathrm{CH}_{4}$ production estimates, developed using the data set derived from 40 crossover studies in dairy cows

\begin{tabular}{|c|c|c|c|c|c|}
\hline Item $^{1}$ & Estimate & $\mathrm{SE}$ & $P>\mathrm{Z}^{2}$ & $\mathrm{SD}^{3}$ & $\mathrm{CV}^{4}$ \\
\hline \multicolumn{6}{|c|}{$\mathrm{CH}_{4} \mathrm{VFA}, \mathrm{mmol} / \mathrm{mol}$ of $\mathrm{VFA}, \mathrm{n}=636$} \\
\hline $\operatorname{Diet}(\operatorname{Exp})$ & 60.5 & 11.85 & $<0.001$ & 7.78 & 0.022 \\
\hline Cow (Exp) & 12.1 & 5.52 & 0.014 & 3.48 & 0.010 \\
\hline Residual & 104.2 & 9.18 & $<0.001$ & 10.21 & 0.028 \\
\hline \multicolumn{6}{|c|}{ Total $\mathrm{CH}_{4}, \mathrm{~g} / \mathrm{d}, \mathrm{n}=360$} \\
\hline $\operatorname{Diet}(\operatorname{Exp})$ & 978 & 304.9 & $<0.001$ & 31.3 & 0.078 \\
\hline Cow (Exp) & 1,745 & 422.1 & $<0.001$ & 41.8 & 0.104 \\
\hline Residual & 2,446 & 330.3 & $<0.001$ & 49.5 & 0.123 \\
\hline \multicolumn{6}{|c|}{$\mathrm{CH}_{4}$ yield, $\mathrm{g} / \mathrm{kg}, \mathrm{n}=360$} \\
\hline $\operatorname{Diet}(\operatorname{Exp})$ & 0.79 & 0.518 & 0.063 & 0.889 & 0.039 \\
\hline Cow (Exp) & 2.26 & 0.750 & 0.001 & 1.502 & 0.067 \\
\hline Residual & 6.97 & 0.905 & $<0.001$ & 2.640 & 0.117 \\
\hline \multicolumn{6}{|c|}{$\mathrm{CH}_{4}-\mathrm{Yan}, \mathrm{g} / \mathrm{d}, \mathrm{n}=360$} \\
\hline $\operatorname{Diet}(\operatorname{Exp})$ & 129 & 31.7 & $<0.001$ & 11.4 & 0.029 \\
\hline Cow (Exp) & 741 & 130.2 & $<0.001$ & 27.2 & 0.070 \\
\hline Residual & 193 & 24.3 & $<0.001$ & 13.9 & 0.035 \\
\hline \multicolumn{6}{|c|}{$\mathrm{CH}_{4}-\mathrm{RH}, \mathrm{g} / \mathrm{d}, \mathrm{n}=360$} \\
\hline $\operatorname{Diet}(\operatorname{Exp})$ & 332 & 71.3 & $<0.001$ & 18.2 & 0.048 \\
\hline Cow (Exp) & 950 & 168.7 & $<0.001$ & 30.8 & 0.081 \\
\hline Residual & 270 & 35.1 & $<0.001$ & 16.4 & 0.043 \\
\hline
\end{tabular}

${ }^{1} \operatorname{Exp}=$ experiment; $\operatorname{Diet}(\operatorname{Exp})=$ diet within experiment; $\operatorname{Cow}(\operatorname{Exp})=$ cow within experiment; $\mathrm{CH}_{4^{-}}$Yan $=$ Equation [5] to predict $\mathrm{CH}_{4}$ production according to Yan et al. (2000); $\mathrm{CH}_{4}-\mathrm{RH}=$ Equation [6] to predict $\mathrm{CH}_{4}$ production according to Ramin and Huhtanen (2013).

${ }^{2}$ Probability of Z-value.

${ }^{3}$ Calculated as the square root of the variance-component estimate.

${ }^{4}$ Calculated as SD divided by the respective mean value of the variable.

Table 7. Variance component estimates of variables related to $\mathrm{N}$ metabolism, developed using the data set derived from 40 crossover studies in dairy cows

\begin{tabular}{|c|c|c|c|c|c|}
\hline Item $^{1}$ & Estimate & $\mathrm{SE}$ & $P>\mathrm{Z}^{2}$ & $\mathrm{SD}^{3}$ & $\mathrm{CV}^{4}$ \\
\hline \multicolumn{6}{|c|}{$\mathrm{RAN}, \mathrm{mg} / \mathrm{dL}, \mathrm{n}=617$} \\
\hline $\operatorname{Diet}(\operatorname{Exp})$ & 2.91 & 0.523 & $<0.001$ & 1.705 & 0.183 \\
\hline $\operatorname{Cow}(\operatorname{Exp})$ & 1.94 & 0.376 & $<0.001$ & 1.391 & 0.149 \\
\hline Residual & 3.53 & 0.321 & $<0.001$ & 1.878 & 0.201 \\
\hline \multicolumn{6}{|c|}{$\mathrm{BCVFA}, \mathrm{mmol} / \mathrm{mol}, \mathrm{n}=531$} \\
\hline $\operatorname{Diet}(\operatorname{Exp})$ & 2.62 & 0.569 & $<0.001$ & 1.618 & 0.123 \\
\hline Cow (Exp) & 2.35 & 0.536 & $<0.001$ & 1.533 & 0.116 \\
\hline Residual & 5.17 & 0.503 & $<0.001$ & 2.273 & 0.173 \\
\hline \multicolumn{6}{|c|}{ Microbial N, g/d, n $=404$} \\
\hline $\operatorname{Diet}(\operatorname{Exp})$ & 412 & 109.7 & $<0.001$ & 20.3 & 0.070 \\
\hline Cow (Exp) & 952 & 192.8 & $<0.001$ & 30.9 & 0.106 \\
\hline Residual & 918 & 105.9 & $<0.001$ & 30.3 & 0.104 \\
\hline \multicolumn{6}{|c|}{ Microbial N, $\mathrm{g} / \mathrm{kg}$ of DMI, $\mathrm{n}=404$} \\
\hline $\operatorname{Diet}(\operatorname{Exp})$ & 0.75 & 0.206 & $<0.001$ & 0.863 & 0.055 \\
\hline Cow (Exp) & 0.37 & 0.153 & 0.008 & 0.606 & 0.039 \\
\hline Residual & 1.85 & 0.213 & $<0.001$ & 1.361 & 0.087 \\
\hline \multicolumn{6}{|c|}{ Microbial N, $\mathrm{g} / \mathrm{kg}$ of OMTDR, $\mathrm{n}=380$} \\
\hline Diet(Exp) & 1.34 & 0.542 & 0.007 & 1.156 & 0.049 \\
\hline Cow (Exp) & 3.36 & 0.850 & $<0.001$ & 1.832 & 0.078 \\
\hline Residual & 6.46 & 0.751 & $<0.001$ & 2.541 & 0.108 \\
\hline
\end{tabular}

${ }^{1} \mathrm{RAN}=$ rumen ammonia $\mathrm{N}$; BCVFA $=$ branched-chain VFA (isobutyrate + isovalerate); OMTDR $=$ OM truly digested in the rumen. Exp $=$ experiment; Diet $(\operatorname{Exp})=$ diet within experiment; Cow $(\operatorname{Exp})=$ cow within experiment.

${ }^{2}$ Probability of Z-value.

${ }^{3}$ Calculated as the square root of the variance component estimate.

${ }^{4}$ Calculated as SD divided by the respective mean value of the variable. 
Table 8. Variance component estimates of rumen pool and digestion kinetics metabolism variables, developed using the data set derived from 40 crossover studies in dairy cows

\begin{tabular}{|c|c|c|c|c|c|}
\hline Item $^{1}$ & Estimate & $\mathrm{SE}$ & $P>\mathrm{Z}^{2}$ & $\mathrm{SD}^{3}$ & $\mathrm{CV}^{4}$ \\
\hline \multicolumn{6}{|c|}{ DM pool, $\mathrm{g} / \mathrm{kg}$ of $\mathrm{BW}, \mathrm{n}=217$} \\
\hline $\operatorname{Diet}(\operatorname{Exp})$ & 0.82 & 0.317 & 0.005 & 0.905 & 0.049 \\
\hline Cow (Exp) & 5.82 & 1.329 & $<0.001$ & 2.413 & 0.131 \\
\hline Residual & 1.78 & 0.292 & $<0.001$ & 1.335 & 0.073 \\
\hline \multicolumn{6}{|c|}{ NDF pool, $\mathrm{g} / \mathrm{kg}$ of $\mathrm{BW}, \mathrm{n}=217$} \\
\hline $\operatorname{Diet}(\operatorname{Exp})$ & 0.80 & 0.225 & $<0.001$ & 0.895 & 0.088 \\
\hline Cow (Exp) & 1.74 & 0.404 & $<0.001$ & 1.321 & 0.130 \\
\hline Residual & 0.69 & 0.111 & $<0.001$ & 0.832 & 0.082 \\
\hline \multicolumn{6}{|c|}{ pdNDF-kd, \%/h, n = 154} \\
\hline $\operatorname{Diet}(\operatorname{Exp})$ & 0.355 & 0.1184 & 0.001 & 0.5961 & 0.124 \\
\hline $\operatorname{Cow}(\operatorname{Exp})$ & 0.313 & 0.1069 & 0.002 & 0.5595 & 0.116 \\
\hline Residual & 0.313 & 0.0577 & $<0.001$ & 0.5596 & 0.116 \\
\hline \multicolumn{6}{|c|}{ pdNDF-kp, $\% / \mathrm{h}, \mathrm{n}=154$} \\
\hline $\operatorname{Diet}(\operatorname{Exp})$ & 0.011 & 0.0062 & 0.041 & 0.1036 & 0.078 \\
\hline Cow(Exp) & 0.010 & 0.0059 & 0.039 & 0.1020 & 0.077 \\
\hline Residual & 0.043 & 0.0079 & $<0.001$ & 0.2083 & 0.157 \\
\hline \multicolumn{6}{|c|}{ iNDF-kp, $\% / \mathrm{h}, \mathrm{n}=154$} \\
\hline $\operatorname{Diet}(\operatorname{Exp})$ & 0.048 & 0.0188 & 0.005 & 0.2202 & 0.086 \\
\hline $\operatorname{Cow}(\operatorname{Exp})$ & 0.053 & 0.0193 & 0.003 & 0.2303 & 0.090 \\
\hline Residual & 0.085 & 0.0153 & $<0.001$ & 0.2909 & 0.113 \\
\hline
\end{tabular}

${ }^{1} \operatorname{Exp}=$ experiment; Diet $(\operatorname{Exp})=$ diet within experiment; $\operatorname{Cow}(\operatorname{Exp})=$ cow within experiment. pdNDF-kd $=$ digestion rate of potentially digestible NDF; pdNDF-kp = passage rate of potentially digestible NDF; iNDF-kd = passage rate of indigestible NDF.

${ }^{2}$ Probability of Z-value.

${ }^{3}$ Calculated as the square root of the variance component estimate.

${ }^{4}$ Calculated as SD divided by the respective mean value of the variable.

proportion of propionate negatively related to OMD, whereas the effect of rumen $\mathrm{pH}$ was nonsignificant. A positive relationship $(P<0.01)$ was observed between OMD and RAN concentration, but OMD decreased $(P$ $<0.01$ ) with increased microbial $\mathrm{N}$ flow and efficiency of microbial $\mathrm{N}$ synthesis.

Rumen ammonia $\mathrm{N}$ concentration was positively $(P$ $<0.01)$ associated with molar proportion of BCVFA and digestion rate of pdNDF, whereas RAN decreased $(P<0.01)$ with increased passage rate of iNDF. In addition, RAN was positively $(P<0.01)$ related to molar proportions of butyrate and butyrate:propionate ratio and negatively $(P<0.01)$ related to molar proportion of propionate (models not shown).

\section{DISCUSSION}

The observed variation in the data set covered the expected ranges in dietary chemical composition, feed intake, and milk production of dairy cow diets in the Nordic countries. In the present study, repeatability and between-cow variation estimated by variance components were used to identify suitable animal variables of nutrient digestion and rumen fermentation related to between-cow differences in $\mathrm{CH}_{4}$ emissions. The effects of diet and period within study were statistically removed from the data so that differences in ruminal digestion relating to variation in $\mathrm{CH}_{4}$ emissions of individual animals could be identified.

Table 9. Influence of animal variables on stoichiometry $\mathrm{CH}_{4}$, estimated by univariate mixed model regression analysis $\left(\mathrm{CH}_{4} \mathrm{VFA}=\mathrm{A}+\mathrm{BX}_{1}\right)$ of the meta-analysis data set derived from 40 crossover studies in dairy cows ${ }^{1}$

\begin{tabular}{lcllrrrrr}
\hline $\mathrm{X}_{1}$ & $\mathrm{n}$ & Units & $\mathrm{A}^{2}$ & $\mathrm{SE}$ & $\mathrm{B}$ & $\mathrm{SE}$ & $P$-value & Residual \\
\hline DMI & 637 & $\mathrm{~kg} / \mathrm{d}$ & 376 & 3.7 & -0.95 & 0.190 & $<0.01$ & 115 \\
Rumen $\mathrm{pH}$ & 597 & $\mathrm{pH}$ & 341 & 13.9 & 2.64 & 2.188 & 0.23 & 121 \\
OMD & 558 & $\mathrm{~g} / \mathrm{kg}$ & 315 & 14.4 & 0.06 & 0.019 & $<0.01$ & 99 \\
NDFD & 542 & $\mathrm{~g} / \mathrm{kg}$ & 334 & 7.0 & 0.04 & 0.011 & $<0.01$ & 103 \\
RAN & 617 & $\mathrm{mg} / \mathrm{dL}$ & 352 & 1.7 & 0.68 & 0.153 & $<0.01$ & 114 \\
BCVFA & 531 & $\mathrm{mmol} / \mathrm{mol}$ & 353 & 3.4 & 0.31 & 0.152 & 0.05 & 125 \\
\hline
\end{tabular}

${ }^{1} \mathrm{OMD}=\mathrm{OM}$ digestibility; NDFD $=$ NDF digestibility; RNDFD = rumen NDF digested; RAN = rumen ammonia $\mathrm{N} ; \mathrm{BCVFA}=$ branched-chain VFA (isobutyrate + isovalerate). $\mathrm{A}=$ intercept; $\mathrm{B}=$ regression coefficient; $\mathrm{X}_{1}=$ variable.

${ }^{2}$ All $P$-values $\leq 0.01$. 


\section{Predicted $\mathrm{CH}_{4}$ and Between-Cow Variation}

Mean predicted $\mathrm{CH}_{4}$ production was in good agreement with the values predicted using empirical models developed from large data sets obtained using respiration chamber studies (Yan et al., 2000; Ramin and Huhtanen, 2013). Predicted $\mathrm{CH}_{4}$ yield was slightly greater (22.6 vs. $21.2 \mathrm{~g} / \mathrm{kg}$ of DM) than in the study by Brask et al. (2015). The difference can be partly related to the dietary greater proportion of corn silage in that study, where $\mathrm{CH}_{4}$ production was predicted from nutrient digestion and fermentation stoichiometry. In the current data set, digesta flow from the rumen was determined by the triple-marker method using ${ }^{15} \mathrm{~N}$ as a marker for microbial protein, which can be considered state-of-the-art methodology. However, random errors in estimating OMADR cause even greater errors in estimates of $\mathrm{CH}_{4}$ production because overestimation of OMADR results in underestimation of the contribution of microbial cells as a $\mathrm{H}_{2}$ sink and vice versa. Without considering microbial cells as a $\mathrm{H}_{2}$ sink (Czerkawski, 1986; Russell, 2002), $\mathrm{CH}_{4}$ production calculated from nutrient digestion and fermentation balance had been clearly overestimated. Ramin and Huhtanen (2013) calculated that the contribution of microbial cells as a $\mathrm{H}_{2}$ sink can account for about $20 \%$ of reduced $\mathrm{CH}_{4}$ yield with increased feed intake. Wolin (1960) approach could be criticized because it assumes that all fermented substrates have a formula $\mathrm{C}_{6} \mathrm{H}_{12} \mathrm{O}_{6}$. Some carbohydrates deviate from this general formula, but they are usually only a small part of ruminant diets (Wolin, 1960). Furthermore, possible deviations from $\mathrm{C}_{6} \mathrm{H}_{12} \mathrm{O}_{6}$ formula could influence variance component of Diet(Exp) but not that of Cow(Exp).
Between-cow coefficients of variation were 0.104 and 0.067 for predicted total $\mathrm{CH}_{4}$ production and $\mathrm{CH}_{4}$ yield, respectively. Between-animal coefficient of variation values in $\mathrm{CH}_{4}$ production reported in the literature vary, reflecting differences in feed intake and methodology. Blaxter and Clapperton (1965) reported coefficient of variation values ranging from 0.072 to 0.081 for $\mathrm{CH}_{4}$ yield by sheep in closed-circuit respiration chambers. Much greater between-cow variation, up to coefficient of variation $=0.30$, has been reported in studies using sniffer methods to determine $\mathrm{CH}_{4}$ emissions (i.e., Garnsworthy et al., 2012; de Haas et al., 2013). In the data set from respiration chamber studies $(\mathrm{n}=539)$ used by Yan et al. (2010), coefficient of variation was 0.17 despite large variation in cow and diet variables $(\mathrm{BW}=$ $379-733 \mathrm{~kg} ; \mathrm{DMI}=7.5-25.0 \mathrm{~kg} / \mathrm{d}$; forage proportion $=$ $18-100 \%)$. This suggests that the sniffer methods exaggerate between-animal differences in $\mathrm{CH}_{4}$ production. Similarly, Huhtanen et al. (2015a) reported greater between-cow variation with the sniffer method than the flux method (GreenFeed system, C-Lock Inc., Rapid City, SD), where the latter is based on spot sampling over several days.

\section{Rumen Fermentation}

Rumen fermentation pattern can be expected to be closely related to $\mathrm{CH}_{4}$ production due to changes in the $\mathrm{H}_{2}$ balance, such that high acetate and butyrate production enhance $\mathrm{CH}_{4}$ production, whereas high propionate production reduces $\mathrm{CH}_{4}$ emissions (Johnson and Johnson, 1995; Moss et al., 2000). Jonker et al. (2016) performed a study in sheep to evaluate the ef-

Table 10. Influence of animal variables on OM digestibility (OMD), estimated by univariate mixed model regression analysis $\left(\mathrm{OMD}=\mathrm{A}+\mathrm{BX}_{1}\right)$ of the meta-analysis data set derived from 40 crossover studies in dairy cows $^{1}$

\begin{tabular}{lclcrrrrr}
\hline $\mathrm{X}_{1}$ & $\mathrm{n}$ & Units & $\mathrm{A}^{2}$ & $\mathrm{SE}$ & $\mathrm{B}$ & $\mathrm{SE}$ & $P$-value & Residual \\
\hline DMI & 637 & $\mathrm{~kg} / \mathrm{d}$ & 780 & 7.7 & -2.21 & 0.384 & $<0.01$ & 272 \\
NDFD & 542 & $\mathrm{~g} / \mathrm{kg}$ & 457 & 10.0 & 0.44 & 0.015 & $<0.01$ & 110 \\
RNDFD/NDFD & 404 & $\mathrm{ratio}$ & 691 & 13.0 & 55.4 & 14.37 & $<0.01$ & 270 \\
pdNDF-kd & 154 & $\% / \mathrm{h}$ & 719 & 10.4 & 4.70 & 1.794 & 0.01 & 280 \\
pdNDF-kp & 154 & $\% / \mathrm{h}$ & 800 & 9.6 & -44.8 & 5.92 & $<0.01$ & 211 \\
Rumen pH & 597 & $\mathrm{pH}$ & 756 & 26.9 & -3.09 & 4.211 & 0.49 & 280 \\
Acetate & 636 & $\mathrm{mmol} / \mathrm{mol}$ & 617 & 39.0 & 0.18 & 0.06 & $<0.01$ & 274 \\
Propionate & 636 & $\mathrm{mmol} / \mathrm{mol}$ & 779 & 11.7 & -0.23 & 0.063 & $<0.01$ & 275 \\
BCVFA & 531 & $\mathrm{mmol} / \mathrm{mol}$ & 729 & 7.3 & 0.51 & 0.333 & 0.13 & 296 \\
RAN & 617 & $\mathrm{mg} / \mathrm{dL}$ & 723 & 4.1 & 1.74 & 0.346 & $<0.01$ & 268 \\
Microbial N & 404 & $\mathrm{~g} / \mathrm{d}$ & 787 & 6.5 & -0.15 & 0.019 & $<0.01$ & 245 \\
Microbial N/kg of OMTDR & 380 & $\mathrm{~g} / \mathrm{kg}$ & 798 & 7.5 & -2.29 & 0.282 & $<0.01$ & 257 \\
\hline
\end{tabular}

${ }^{1} \mathrm{NDFD}=\mathrm{NDF}$ digestibility; RNDFD = rumen NDF digested; pdNDF-kd = digestion rate of potentially digestible NDF (pdNDF); pdNDF-kp = passage rate of pdNDF; both acetate and propionate as molar proportions of VFA; $\mathrm{CH}_{4} \mathrm{VFA}=$ stoichiometry $\mathrm{CH}_{4} ; \mathrm{BCVFA}=$ branched-chain VFA (isobutyrate + isovalerate); $\mathrm{RAN}$ $=$ rumen ammonia $\mathrm{N}$; OMTDR $=\mathrm{OM}$ truly digested in the rumen. $\mathrm{A}=$ intercept; $\mathrm{B}=$ regression coefficient; $\mathrm{X}_{1}=$ variable.

${ }^{2}$ All $P$-values $\leq 0.01$. 
fects of graded substitution of alfalfa silage with corn silage or corn grain in a diet fed at a fixed DMI level ( $2 \%$ of $\mathrm{BW}$ ) on rumen fermentation characteristics in vivo and in vitro. They observed a quadratic effect of both supplements on $\mathrm{CH}_{4}$ yield, to a maximum at $50 \%$, and thereafter it decreased more rapidly for the corn grain supplement. The ratio of (acetate + butyrate)/ (propionate + valerate) and the propionate concentration alone were the best single predictors of $\mathrm{CH}_{4}$ yield (Jonker et al., 2016).

In the present study, between-cow variation in rumen fermentation pattern was small $(\mathrm{CV}=0.010$ for stoichiometric $\mathrm{CH}_{4} \mathrm{VFA}$ ), confirming data in Schiemann et al. (1971) where the calculated coefficients of variation of $\mathrm{CH}_{4}$ yield and $\mathrm{CH}_{4} \mathrm{VFA}$ ratio (Wolin, 1960) were 0.082 and 0.014 , respectively, for 8 cows fed at the maintenance level, and 0.090 and 0.013 , respectively, for 8 cows fed at the production level. Pinares-Patiño et al. (2013) found a coefficient of variation for $\mathrm{CH}_{4}$ yield of 0.098 in a study with 10 sheep, whereas the coefficient of variation value for molar proportions of acetate, propionate, and butyrate was $0.011,0.047$, and 0.036 , respectively. Small between-animal variation in rumen fermentation pattern relative to the variation in $\mathrm{CH}_{4}$ yield does not indicate a major direct effect of the rumen microbiome on between-animal differences in $\mathrm{CH}_{4}$ emissions. Indeed, the contribution of betweencow $\mathrm{CH}_{4} \mathrm{VFA}$ variation was considerably smaller than estimated using empirical equations considering feeding level $\left(\mathrm{CH}_{4}-\mathrm{Yan}\right)$ or diet characteristics $\left(\mathrm{CH}_{4}-\mathrm{RH}\right)$.

Ruminal microbiome data have been infrequently associated with data on ruminal fermentation and because methanogenic communities in the rumen can vary in response to diet and animal variables, these results have to be interpreted with caution. In lactating dairy cows with differing residual feed intake (RFI) phenotype, no meaningful differences in rumen microbiome were associated with rumen fermentation pattern in a study by Rius et al. (2012). The absence of differences in the rumen microbiome observed in the Rius et al. (2012) study is in line with the lack of differences in the major VFA observed in the present study. Differences in rumen microbiome structure between different animals within the same diet group often exceed those observed between different diet groups (Firkins and $\mathrm{Yu}$, 2015). Between-animal differences are typically attributed to different animal genetics and host interactions with the rumen microbiome (Weimer et al., 2010). In the study of Roehe et al. (2016) sire progeny groups differed significantly in their $\mathrm{CH}_{4}$ emissions. Ranking of the sire progeny groups based on $\mathrm{CH}_{4}$ emissions or relative archaeal abundance was consistent. This suggested that archaeal abundance in ruminal digesta was under host genetic control as previously reported by Guan et al. (2008).

In the present study, dietary variances in molar proportions of VFA and $\mathrm{CH}_{4} \mathrm{VFA}$ were much greater than the corresponding animal variances, suggesting that rumen fermentation pattern was more strongly associated with differences in fermented substrates between the diets than with differences in rumen microbiome between the cows. It is possible that between-animal variation increases with increased proportion of concentrate. The sum of Cow(Exp) and residual variance of $\mathrm{CH}_{4}$ VFA was about 2 -fold higher (163 vs. 76 ) for diets above, rather than below, $42 \%$ concentrate in diet DM. It was not possible to estimate between-animal variance for diets with $>42 \%$ concentrate because there was not enough replication within the study. A higher betweenanimal coefficient of variation for total $\mathrm{CH}_{4}$ production and $\mathrm{CH}_{4}$ yield for cattle fed a high-concentrate diet compared with a high-forage diet (Herd et al., 2016; Roehe et al., 2016) is in line with this suggestion.

Firkins and $\mathrm{Yu}$ (2006) concluded that abundance of methanogens might not be a reliable indicator of methane emission from ruminants, a claim supported by results of Danielsson et al. (2012). In the study by Jonker et al. (2016), Methanobrevibacter ruminantium and Methanosphera spp. clades had weak to moderate correlations with $\mathrm{CH}_{4}$ yield. Methanogen densities were not significantly different in rumen samples from high and low emitters, but archaeal community structure was significantly different in rumen samples associated with different $\mathrm{CH}_{4}$ yields (Kittelmann et al., 2014). Moreover, high emitters had higher relative abundance of species that are known to form significant amounts of $\mathrm{H}_{2}$, whereas lower $\mathrm{CH}_{4}$ yields were related to bacterial communities that produce relatively less $\mathrm{H}_{2}$. In a study by Carberry et al. (2012), few significant correlations between relative microbial abundance and end products of rumen fermentation or digestibility were observed. The most obvious were negative and positive correlations between relative abundance of protozoa and molar proportions of propionate and butyrate in rumen VFA, respectively. However, it should be borne in mind that in these studies conclusions are often based on statistical associations rather than rigorous demonstration of cause and effect. Part of the inconsistency between $\mathrm{CH}_{4}$ emissions and archaea abundance could be related to within-species expression of genes involved in the methanogenic pathway, as shown by Shi et al. (2014). In their study, the abundance of methanogens and methanogenesis pathway genes were similar between low and high $\mathrm{CH}_{4}$ emitters, but transcription of these genes was substantially higher in high emitters than in low emitters. 
In the present study, repeatability of $\mathrm{CH}_{4} \mathrm{VFA}$ was also low (0.11), probably because of small overall between-cow variation in this trait. Interestingly, repeatability was higher (0.19) for 2 consecutive periods than for all data (0.11), or for the first and last period (0.09). Low repeatability of $\mathrm{CH}_{4} \mathrm{VFA}$ was consistent with the sheep study by Robinson et al. (2010). Pinares-Patiño et al. (2013) found that the repeatability of $\mathrm{CH}_{4}$ yield in sheep measured on consecutive days was high (0.89), but it was only 0.26 for measurements 2 wk apart, and 0.24 across years. These observations suggest that the microbial population in the rumen can alter with time (Welkie et al., 2010; Mullins et al., 2013). Greater between-cow variation and repeatability of traits such as digestibility, passage, and efficiency of microbial cell synthesis indicate that between-animal variation in $\mathrm{CH}_{4}$ yield may be more closely related to these characteristics than the composition of the rumen microbiome. It is important to emphasize that $\mathrm{CH}_{4} \mathrm{VFA}$ does not take into account digestibility or passage rate through the gastrointestinal tract and only reflects the VFA fermentation profile in the rumen.

Between-cow variation was smaller when predicted by empirical models (Yan et al., 2000; Ramin and Huhtanen, 2013) than when predicted from the amount of OMADR using stoichiometric principles in the present study. This is because those empirical models do not take into account the contribution of digestibility and rumen fermentation pattern in the estimated value of the Cow $(\operatorname{Exp})$ variance component. The greater between-cow variation obtained with the model of Ramin and Huhtanen (2013) compared with that of Yan et al. (2000) is most likely because the former takes variation in diet digestibility and composition into account. Both empirical models are based on in vivo $\mathrm{CH}_{4}$ measurements made in respiration chambers on animals fed grass silage.

\section{Digestibility}

The between-cow variation in OMD was small $(\mathrm{SD}=10 \mathrm{~g} / \mathrm{kg} ; \mathrm{CV}=0.013)$. Mehtiö et al. (2016) reported a value of $12.3 \mathrm{~g} / \mathrm{kg}$ for between-cow variation in OMD determined using acid-insoluble ash as an internal marker. Greater variability was reported by Berry et al. (2007) for grazing cows when digestibility was determined using alkanes as a marker, probably because that study used only one replicate per animal (SD represents between-cow and random variation). In grazing conditions, plant selection can also increase the between-cow variation in digestibility. Mean retention time (MRT; reciprocal of passage rate) of the digesta is probably the main contributor to between-cow variation in digestibility. Ørskov et al. (1988) reported a strong negative $(-0.80)$ correlation coefficient between passage rate of $\mathrm{Cr}$-mordanted straw and digestibility for 22 cows. On average, OMD increased by $4.9 \mathrm{~g} / \mathrm{kg}$ per $1 \mathrm{~h}$ increase in MRT. In the modeling study by Huhtanen et al. (2016), the corresponding response in OMD was $3.9 \mathrm{~g} / \mathrm{kg}$ per $1 \mathrm{~h}$ increase in MRT. Predicted between-cow variation in OMD was $12.0 \mathrm{~g} / \mathrm{kg}$ for 100 simulations using the variation in MRT of iNDF observed in the present study $(\mathrm{CV}=0.085)$.

Overall, between-cow variation in digestibility can explain only a small part of the estimated variation in $\mathrm{CH}_{4}$ production. Analysis of the data from respiration chamber studies (Yan et al., 2000, 2010; Ramin and Huhtanen, 2013) indicates that $\mathrm{CH}_{4}$ yield decreases by about $10 \%$ per multiple of maintenance increase in feeding level. This effect is much greater than observed decreases of approximately 2 to $3 \%$ in OMD per multiple of maintenance (Yan et al., 2002; Huhtanen et al., 2009; i.e., reduced digestibility can only partly explain lower $\mathrm{CH}_{4}$ yield with increased intake). However, it is possible that incremental digestibility produces more $\mathrm{CH}_{4}$ than the diet on average. Schiemann et al. (1971) published individual data for 8 cows fed either at maintenance or production level, for which there was a strong positive relationship between diet digestibility and $\mathrm{CH}_{4}$ yield in both cases. In both the respiration chamber study with dairy cows (Schiemann et al., 1971) and in the modeling study (Huhtanen et al., 2016), the slope between digestibility and $\mathrm{CH}_{4}$ yield was about 3 -fold the average $\mathrm{CH}_{4}$ yield, suggesting that incremental digestion produced more $\mathrm{CH}_{4}$ per unit digested $\mathrm{OM}$ than the diet on average. Stoichiometric $\mathrm{CH}_{4}$ ratio was positively related to OMD, but quantitatively the effect was small; a \pm 1 standard deviation variation in OMD was associated with only a $0.34 \%$ difference in $\mathrm{CH}_{4} \mathrm{VFA}$. It is possible that between-cow differences in OMD result mainly from the digestion of the slowly digestible NDF fraction, which can produce more acetate and $\mathrm{CH}_{4}$. Positive relationships of OMD and NDF to molar proportion of acetate, and negative relationships to propionate, support this suggestion.

\section{Passage Rate}

Digesta passage rate from the rumen or its reciprocal, MRT, has been related to between-animal differences in $\mathrm{CH}_{4}$ production. In studies with sheep fed at fixed levels, $\mathrm{CH}_{4}$ emissions were negatively related to fractional outflow rate from the rumen (Pinares-Patiño et al., 2003) and particulate MRT was longer for high emitters compared with low emitters (Pinares-Patiño et al., 2011; Goopy et al., 2014). The simulation study by Huhtanen et al. (2016) also showed a positive relationship between MRT and $\mathrm{CH}_{4}$ production. In the 
present study, the passage rate of iNDF was moderately repeatable (0.38). In a simulation study by Huhtanen et al. (2016), the coefficient of variation of predicted $\mathrm{CH}_{4}$ yield was 0.052 for cows and 0.045 for sheep when the simulations were made using the variation in MRT of iNDF in the current data set. Although predicted variation in $\mathrm{CH}_{4}$ emissions was smaller than in controlled respiration studies, both the present study and the simulation study by Huhtanen et al. (2016) indicate that a considerable proportion of between-animal variation can be related to digestive processes.

Earlier studies with sheep (Faichney, 1993) and cattle (Ørskov et al., 1988) found that the ranking of animals on the basis of rumen fractional passage rate was consistent among diets and feeding levels. Smuts et al. (1995) reported that rumen digesta retention time is a repeatable physiological trait $(\operatorname{Rep}=0.45)$. As a consequence, selecting animals for low $\mathrm{CH}_{4}$ emissions may result in animals with lowered digestion capacity of cell wall carbohydrates. The effects of digesta MRT on $\mathrm{CH}_{4}$ emissions are smaller than the effect on diet digestibility on energy basis. The efficiency of microbial $\mathrm{N}$ synthesis is positively related to feed intake (Chen et al., 1992; Broderick et al., 2010). Increased intake would increase ruminal passage rate (i.e., NRC, 2001) and reduce microbial retention time, and thus increase microbial cell yield per unit energy fermentation by diluting maintenance expenditure (Russell et al., 1992). The relationship between passage rate and the efficiency of microbial $\mathrm{N}$ synthesis could be expected to be similar when the differences in passage rate are derived from differences in feeding level or from between-animal differences. With improved efficiency of microbial synthesis associated with reduced retention time, fermented carbon is repartitioned from VFA and gas production to microbial cells. In addition, because microbial cells are more reduced than dietary carbohydrates (Hungate et al., 1971; Czerkawski, 1986; Van Soest, 1994), the stoichiometric fermentation balance (Wolin, 1960) will overestimate $\mathrm{CH}_{4}$ emissions (Van Soest, 1994; Russell, 2002). A significant cow effect on microbial N flow and efficiency of synthesis and moderate repeatability of these characteristics suggest that the traits related to the efficiency of microbial growth in the rumen contribute to between-cow variation in $\mathrm{CH}_{4}$ emissions.

Studies using either empirical equations (Ramin and Huhtanen, 2013) or a mechanistic modeling approach (Huhtanen et al., 2015b) indicate that improved efficiency of microbial protein synthesis makes an important contribution to reduced $\mathrm{CH}_{4}$ yield with increased DMI. De novo fatty acid synthesis would be an important sink for carbon diverted from fermentation, but also for reducing equivalents derived from fermentation (Hackmann and Firkins, 2015).

\section{Relationships Between Variables Affecting $\mathrm{CH}_{4}$ Production}

Several traits related to $\mathrm{CH}_{4}$ production varied significantly between the cows and most of these were moderately repeatable. However, overall effects of these traits on $\mathrm{CH}_{4}$ production depend on their mutual relationships. Diet digestibility could be expected to be positively related to $\mathrm{CH}_{4}$ yield, because more substrate is fermented in the rumen. Improved OMD was positively associated with the molar proportion of acetate and $\mathrm{CH}_{4} \mathrm{VFA}$ and negatively with the molar proportion of propionate. Thus, the effects of OMD and $\mathrm{CH}_{4} \mathrm{VFA}$ are additive. Because most of the variation in OMD was due to NDFD (cow variance for NDFD was 3 -fold that for digestibility of $\mathrm{ND}$ solubles) and $\mathrm{CH}_{4} \mathrm{VFA}$ ratio was positively related to dietary NDF concentration, incremental digestibility increased $\mathrm{CH}_{4} \mathrm{VFA}$.

Between-cow variation in digestibility can result from changes in digestion and passage kinetic parameters, changes in the rumen microbial population, or both. In the present study, passage rate of pdNDF predicted OMD better than digestion rate of pdNDF (residual variance 209 and 279 , respectively). This suggests that variation in digestibility is related more to physiological traits of the animal regulating passage rate, rather than the rumen microbiome regulating digestion rate. The differences in $\mathrm{CH}_{4}$ yield between low- and high-emitting sheep have been associated with digesta retention time (Pinares-Patiño et al., 2003, 2011; Goopy et al., 2014), with diet digestibility being significantly (PinaresPatiño et al., 2011) or numerically (Goopy et al., 2014) lower in low-emitting sheep. Methane yield and OMD were higher in low-RFI (efficient) steers than high-RFI steers in a study by McDonnell et al. (2016). However, the differences in diet digestibility between low and high emitters could only partly explain the difference in $\mathrm{CH}_{4}$ yield.

The effects of the rumen microbiome on diet digestibility could derive from increased potential NDFD, digestion rate of pdNDF, or both. In our in-house determinations of iNDF concentration by 12-d intraruminal in situ incubation, the between-cow variation was small compared with the observed between-cow variation in OMD (unpublished data), suggesting that the effects of the rumen microbiome on the potential extent of fiber digestion are small. Carberry et al. (2012) reported a negative correlation between relative abundance of Prevotella sp. and OMD. In the study by Rius et al. (2012), low-RFI cows had higher OMD than high-RFI cows, but rumen microbiome was not related to digestibility. Ample evidence indicates that cell wall degradation follows first-order kinetics with respect to substrate concentration (Waldo et al., 1972; Van Soest, 
1973), and that the rate is limited by the characteristics of cell walls rather than the abundance or activity of the microbial population. In fact, in vitro studies have revealed that digestion rate of NDF does not decline until ruminal contents are diluted about 6-fold (Pell and Schofield, 1993; Mouriño et al., 2001).

Interestingly, despite significant cow variance and moderate repeatability of rumen $\mathrm{pH}$, this trait was not significantly related to OMD or NDFD. It is possible that with dairy cow diets with mean rumen $\mathrm{pH}$ (6.4), between-cow variation $(\mathrm{SD}=0.13)$ is not large enough to influence digestibility. In a rumen content exchange experiment by Weimer et al. (2010), ruminal pH and VFA profiles returned to those of the recipient cows much more quickly (within $1 \mathrm{~d}$ ) than did bacterial community composition, indicating that the animal exerts ultimate control over its ruminal chemistry through salivary buffering and through absorption and passage of VFA.

The efficiency of microbial $\mathrm{N}$ synthesis, expressed as microbial N per kilogram of DMI, digested OM intake, or OM truly digested in the rumen, was negatively related to OMD or NDFD and positively to passage rate of pdNDF. This indicates that the effects of digestibility and microbial efficiency on $\mathrm{CH}_{4}$ production are additive. Both digestibility and microbial efficiency are related to passage rate; digestibility, especially that of the plant cell wall fraction, decreases with increased passage rate and microbial efficiency improves, as less ATP is required for microbial maintenance. Methane production can be expected to decrease with increased passage rate because (1) less substrate is fermented, and (2) there is repartition of fermented carbon from VFA and gas production to microbial cells, (3) which in turn are more reduced than fermented carbohydrates. The efficiency of microbial synthesis was negatively correlated with molar proportions of BCVFA in the rumen. Branched-chain VFA are produced in the deamination of branched-chain AA and the changes in their concentrations reflect a balance between degradation and utilization for microbial cell synthesis (Russell and Sniffen, 1984; Bach et al., 2005).

\section{CONCLUSIONS}

The present analysis showed that variables related to animal physiology, such as variation in digesta retention time, can explain most of the between-animal variation in $\mathrm{CH}_{4}$ yield. Only small variations were observed in rumen fermentation variables, especially stoichiometric $\mathrm{CH}_{4} \mathrm{VFA}$, suggesting a minor contribution of the rumen microbiome to $\mathrm{CH}_{4}$ production. The variation in nutrient digestibility was also small and therefore did not account for much of the variation in $\mathrm{CH}_{4}$ yield.
However, the variation in digestibility can be attributed at least partly to differences in digesta passage rate, which influence the efficiency of microbial cell synthesis in repartitioning fermented carbon between VFA, gas production, and microbial cells. If between-animal variation in $\mathrm{CH}_{4}$ yield is related to digesta passage rate, care should be exercised when selecting low-emitting animals in breeding programs, as this could lead to reduced efficiency of cell wall digestion.

\section{ACKNOWLEDGMENTS}

The authors gratefully acknowledge the contributions of colleagues from Agri-Food Research in Finland (currently Natural Resources Institute Finland-LUKE, Jokioinen, Finland), Swedish University of Agricultural Sciences (Uppsala, Sweden), and Norwegian University of Life Sciences (Ås, Norway), for providing the data to complete the individual cow data set. This study was supported financially by the Swedish Research Council-FORMAS (project no. 220-2011-1247).

\section{REFERENCES}

Ahvenjärvi, S., A. Vanhatalo, P. Huhtanen, and T. Varvikko. 2000 Determination of reticulo-rumen and whole-stomach digestion in lactating cows by omasal canal or duodenal sampling. Br. J. Nutr. 83:67-77.

Bach, A., S. Calsamiglia, and M. D. Stern. 2005. Nitrogen metabolism in the rumen. J. Dairy Sci. 88(E Suppl.):E9-E21.

Belsley, D. A., E. Kuh, and R. E. Welsch. 1980. Regression Diagnostics: Identifying Influential Data and Sources of Collinearity. John Wiley and Sons Inc., New York, NY.

Berry, D. P., B. Horan, M. O'Donovan, F. Buckley, E. Kennedy, M. McEvoy, and P. Dillon. 2007. Genetics of grass dry matter intake, energy balance, and digestibility in grazing Irish dairy cows. J. Dairy Sci. 90:4835-4845.

Blaxter, K. L., and J. L. Clapperton. 1965. Prediction of the amount of methane produced by ruminants. Br. J. Nutr. 19:511-522.

Brask, M., M. R. Weisbjerg, A. L. F. Hellwing, A. Bannink, and P. Lund. 2015. Methane production and diurnal variation measured in dairy cows and predicted from fermentation pattern and nutrient or carbon flow. Animal 9:1795-1806.

Broderick, G. A., P. Huhtanen, S. Ahvenjärvi, S. M. Reynal, and K. J. Shingfield. 2010. Quantifying ruminal nitrogen metabolism using the omasal sampling technique in cattle-A meta-analysis. J. Dairy Sci. 93:3216-3230.

Carberry, C. A., D. A. Henny, S. Han, M. S. McCabe, and S. M. Waters. 2012. Effect of phenotypic residual feed intake and dietary forage content on the rumen microbial community of beef cattle. Appl. Environ. Microbiol. 78:4949-4958.

Chen, X. B., Y. K. Chen, M. F. Franklin, and E. R. Ørskov. 1992 The effect of feed intake and body weight on purine derivative excretion and microbial protein supply in sheep. J. Anim. Sci. 70:1534-1542.

Czerkawski, J. W. 1986. An Introduction to Rumen Studies. Robert Maxwell, M. C., Oxford, UK.

Danielsson, R., A. Schnürer, V. Arthurson, and J. Bertilsson. 2012. Methanogenic population and $\mathrm{CH}_{4}$ production in Swedish dairy cows fed different levels of forage. Appl. Environ. Microbiol. 78:6172-6179.

de Haas, Y., J. W. van Riel, R. F. Veerkamp, W. Liansun, and N. Ogink. 2013. On-farm methane measurements in exhaled air of in- 
dividual Dutch cows obtained during milking using Fourier transformed infrared methods. Adv. Anim. Biosci. 4:391.

Ellis, J. L., E. Kebreab, N. E. Odongo, B. W. Mcbride, E. K. Okine, and J. France. 2007. Prediction of methane production from dairy and beef cattle. J. Dairy Sci. 90:3456-3466.

Ellis, W. C., J. H. Matis, T. M. Hill, and M. R. Murphy. 1994. Methodology for estimating digestion and passage kinetics of forages. Pages 682-756 in Forage Quality, Evaluation, and Utilization. G. C. Fahey, Jr., M. Collins, D. R. Mertens, and L. E. Moser, ed. Am. Soc. Agron., Madison, WI.

Faichney, G. J. 1993. Digesta flow. Pages 53-85 in Quantitative Aspects of Ruminant Digestion and Metabolism. J. M. Forbes and J. France, ed. CAB Int., Wallingford, UK.

Firkins, J. L., and Z. Yu. 2006. Characterisation and quantification of the microbial populations in the rumen. Pages 19-54 in Ruminant Physiology, Digestion, Metabolism and Impact of Nutrition on Gene Expression, Immunology and Stress. K. Sejrsen, T. Hvelplund, and M. O. Nielsen, ed. Wageningen Acad., Wageningen, the Netherlands.

Firkins, J. L., and Z. Yu. 2015. Ruminant Nutrition Symposium-How to use data on the rumen microbiome to improve our understanding of ruminant nutrition. J. Anim. Sci. 93:1450-1470.

France, J., and R. C. Siddons. 1986. Determination of digesta flow by continuous marker infusion. J. Theor. Biol. 121:105-120.

Garnsworthy, P. C., J. Craigon, J. H. Hernandez-Medrano, and N. Saunders. 2012. On-farm methane measurements during milking correlate with total methane production by individual dairy cows. J. Dairy Sci. 95:3166-3180.

Goopy, J. P., A. Donaldson, R. Hegarty, P. E. Vercoe, F. Haynes, M. Barnett, and V. H. Oddy. 2014. Low-methane yield sheep have smaller rumens and shorter rumen retention time. Br. J. Nutr. 111:578-585

Guan, L. L., D. J. Nkrumah, J. A. Basarab, and S. S. Moore. 2008. Linkage of microbial ecology to phenotype: correlation of rumen microbial ecology to cattle's feed efficiency. FEMS Microbiol. Lett. 288:85-91.

Hackmann, T. J., and J. L. Firkins. 2015. Maximizing efficiency of rumen microbial protein production. Front. Microbiol. 6:465.

Herd, R. M., J. I. Velazco, P. F. Arthur, and R. F. Hegarthy. 2016. Associations among methane emission traits measured in the feedlot and in respiration chambers in Angus cattle bred to vary in feed efficiency. J. Anim. Sci. 94:4882-4891.

Hristov, A. N., J. Oh, J. L. Firkins, J. Dijkstra, J. E. Kebreab, G. Waghorn, H. P. S. Makkar, A. T. Adesogan, W. Yang, C. Lee, P. J. Gerber, B. Henderson, and J. M. Tricarico. 2013. Special TopicsMitigation of methane and nitrous oxide emissions from animal operations: I. A review of enteric methane mitigation options. J. Anim. Sci. 91:5045-5069.

Huhtanen, P., E. H. Cabezas-Garcia, S. Utsumi, and S. Zimmerman. 2015a. Comparison of methods to determine methane emissions from dairy cows in farm conditions. J. Dairy Sci. 98:3394-3409.

Huhtanen, P., K. Kaustell, and S. Jaakkola. 1994. The use of internal markers to predict total digestibility and duodenal flow of nutrients in cattle given six different diets. Anim. Feed Sci. Technol. 48:211-227.

Huhtanen, P., M. Ramin, and E. H. Cabezas-Garcia. 2016. Effects of ruminal digesta retention time on methane emissions: A modelling approach. Anim. Prod. Sci. 56:501-506.

Huhtanen, P., M. Ramin, and P. Udén. 2015b. Nordic dairy cow model Karoline in predicting methane emissions: 1. Model description and sensitivity analysis. Livest. Sci. 178:71-80.

Huhtanen, P., M. Rinne, and J. Nousiainen. 2009. A meta-analysis of feed digestion in dairy cows. 2 . The effects of feeding level and diet composition on digestibility. J. Dairy Sci. 92:5031-5042.

Hungate, R. E. 1966. The Rumen and its Microbes. Academic Press, New York, NY.

Hungate, R. E., J. Reichl, and R. A. Prins. 1971. Parameters of rumen fermentation in a continuously fed sheep: Evidence of a microbial rumination pool. Appl. Microbiol. 22:1104-1113.

Johnson, K. A., and D. E. Johnson. 1995. Methane emissions from cattle. J. Anim. Sci. 73:2483-2492.
Jonker, A., K. Lowe, S. Kittelmann, P. H. Janssen, S. Ledgard, and D. Pacheco. 2016. Methane emissions changed nonlinearly with graded substitution of alfalfa silage with corn silage and corn grain in the diet of sheep and relation with rumen fermentation characteristics in vivo and in vitro. J. Anim. Sci. 94:3464-3475.

Kittelmann, S., C. S. Pinares-Patiño, H. Seedorf, M. R. Kirk, S. Ganesh. J. C. McEwan, and P. H. Janssen. 2014. Two different bacterial community types are linked with the low-methane emission trait in sheep. PLoS One 9:e103171.

Littell, R. C., G. A. Milliken, W. W. Stroup, R. D. Wolfinger, and O. Schabenberger. 2006. SAS for Mixed Models. 2nd ed. SAS Institute Inc., Cary, NC.

LUKE. 2016. Finnish Feed tables. Accessed Jul. 1, 2016. https://portal. mtt.fi/portal/page/portal/Rehutaulukot/feed_tables_english.

McDonnell, R. P., K. J. Hart, T. M. Boland, A. K. Kelly, M. McGee, and D. A. Kenny. 2016. Effect of divergence in phenotypic residual feed intake on methane emissions, ruminal fermentation, and apparent whole-tract digestibility of beef heifers across three contrasting diets. J. Anim. Sci. 94:1179-1193

Mehtiö, T., M. Rinne, L. Nyholm, P. Mäntysaari, A. Sairanen, E. A. Mäntysaari, T. Pitkänen, and M. H. Lidauer. 2016. Cow-specific diet digestibility predictions based on near-infrared reflectance spectroscopy scans of faecal samples. J. Anim. Breed. Genet. 133:115-125.

Moss, A. R., J. P. Jouany, and J. Newbold. 2000. Methane production by ruminants: Its contribution to global warming. Ann. Zootech. 49:231-253.

Mouriño, F., R. Akkarawongsa, and P. J. Weimer. 2001. Initial pH as a determinant of cellulose digestion rate by mixed ruminal microorganisms in vitro. J. Dairy Sci. 84:848-859.

Mullins, C. R., L. K. Mamedova, A. J. Carpenter, Y. Ying, M. S. Allen, I. Yoon, and B. J. Bradford. 2013. Analysis of rumen microbial populations in lactating dairy cattle fed diets varying in carbohydrate profiles and Saccharomyces cerevisiae fermentation product. J. Dairy Sci. 96:5872-5881.

NRC. 2001. Nutrient Requirements of Dairy Cattle. 7th rev. ed. National Academy Press, Washington, DC.

Ørskov, E. R., I. Ojwang, and G. W. Reid. 1988. A study on consistency of differences between cows in rumen outflow rate of fibrous particles and other substrates and consequences for digestibility and intake of roughages. Anim. Prod. 47:45-51.

Pell, A. N., and P. Schofield. 1993. Computerized monitoring of gas production to measure forage digestion in vitro. J. Dairy Sci 76:1063-1073.

Pinares-Patiño, C. S., S. H. Ebrahimi, J. C. McEwan, H. Clark, and D. Luo. 2011. Is rumen retention time implicated in sheep differences in methane emission? Proc. N.Z. Soc. Anim. Prod. 71:219-222.

Pinares-Patiño, C. S., S. M. Hickey, E. A. Young, K. G. Dodds, S. Maclean, G. Molano, E. Sandoval, H. Kjestrup, R. Harland, C. Hunt, N. K. Pickering, and J. C. McEwan. 2013. Heritability estimates of methane emissions from sheep. Animal 7:316-321.

Pinares-Patiño, C. S., M. J. Ulyatt, K. R. Lassey, T. N. Barry, and C. W. Holmes. 2003. Rumen function and digestion parameters associated with differences between sheep in methane emissions when fed chaffed Lucerne hay. J. Agric. Sci. 140:205-214.

Ramin, M., and P. Huhtanen. 2013. Development of equations for predicting methane emissions from ruminants. J. Dairy Sci. 96:24762493.

Rius, A. G., S. Kittelmann, K. A. Macdonald, G. C. Waghorn, P. H. Janssen, and E. Sikkema. 2012. Nitrogen metabolism and rumen microbial enumeration in lactating cows with divergent residual feed intake fed high-digestibility pasture. J. Dairy Sci. 95:50245034 .

Robinson, D. L., J. P. Goopy, R. S. Hegarty, and P. E. Vercoe. 2010. Repeatability, animal and sire variation in 1-hr methane emissions and relationship with rumen volatile fatty acid concentrations. Abstract no. 712 in Proc. 9th World Congress in Genetics Applied to Livestock. Book of Abstracts. German Society of Animal Science, Leipzig, Germany.

Roehe, R., R. J. Dewhurst, C.-A. Duthie, J. A. Rooke, N. McKain, D. W. Ross, J. J. Hyslop, A. Waterhouse, T. C. Freeman, M. 
Watson, and R. J. Wallace. 2016. Bovine host genetic variation influences rumen microbial methane production with best selection criterion for low methane emitting and efficiently feed converting hosts based on metagenomics gene abundance. PLoS Genet. 12:e1005846.

Russell, J. B. 2002. Rumen Microbiology and its Role in Ruminant Nutrition, 1st ed. Ithaca, NY.

Russell, J. B., J. D. O'Connor, D. G. Fox, P. J. Van Soest, and C. J. Sniffen. 1992. A net carbohydrate and protein system for evaluating cattle diets: I. Ruminal fermentation. J. Anim. Sci. 70:35513561.

Russell, J. B., and C. J. Sniffen. 1984. Effect of carbon-4 and carbon-5 volatile fatty acids on growth of mixed rumen bacteria in vitro. J. Dairy Sci. 67:987-994.

Schiemann, R., W. Jentsch, and H. Wittenburg. 1971. Zur Abhängigkeit der Verdaulichkeit der Energie und der Nährstoffe von der Höhe der Futteraufnahme und der Rationszusammensetzung bei Milchkühen. Arch. Tierernahr. 21:223-240.

Shi, W., C. D. Moon, S. C. Leahy, D. Kang, J. Froula, S. Kittelmann, C. Fan, S. Deutsch, D. Gagic, H. Seedorf, W. J. Kelly, R. Atua, C. Sang, P. Soni, D. Li, C. S. Pinares-Patiño, J. C. McEwan, P. H. Janssen, F. Chen, A. Visel, Z. Wang, G. T. Attwood, and E. M. Rubin. 2014. Methane yield phenotypes linked to differential gene expression in the sheep rumen microbiome. Genome Res. $24: 1517-1525$.

Sjaunja, L. O., L. Baevre, L. Junkkarinen, J. Pedersen, and J. Setälä. 1991. A Nordic proposal for an energy corrected milk (ECM) formula. Pages 156-157 in Recording of Animals - State of the Art 1990. EAAP Publ. 50. Perform. Centre Agric. Publ. Doc. (PUDOC), Wageningen, the Netherlands.

Smuts, M., H. H. Meissner, and P. B. Cronjé. 1995. Retention time of digesta in the rumen: Its repeatability and relationship with wool production of Merino rams. J. Anim. Sci. 73:206-210.

St-Pierre, N. R. 2001. Integrating quantitative findings from multiple studies using mixed model methodology. J. Dairy Sci. 84:741-755.

Van Keulen, J., and B. A. Young. 1977. Evaluation of acid-insoluble ash as a natural marker in ruminant digestibility studies. J. Anim. Sci. 44:282-287.
Van Soest, P. J. 1973. The uniformity and nutritive availability of cellulose. Fed. Proc. 32:1804-1808.

Van Soest, P. J. 1994. Nutritional Ecology of the Ruminant. Cornell University Press, Ithaca, NY.

Vlaming, J. B., N. Lopez-Villalobos, I. M. Brookes, S. O. Hoskin, and H. Clark. 2008. Within- and between-animal variance in methane emissions in non-lactating dairy cows. Aust. J. Exp. Agric. 48:124-127.

Waldo, D. R., L. W. Smith, and E. L. Cox. 1972. Model of cellulose disappearance from the rumen. J. Dairy Sci. 55:125-129.

Weimer, P. J., D. M. Stevenson, H. C. Mantovani, and S. L. C. Man. 2010. Host specificity of the ruminal bacterial community of the dairy cow following near-total exchange of ruminal contents. J. Dairy Sci. 93:5902-5912.

Welkie, D. G., D. M. Stevenson, and P. J. Weimer. 2010. ARISA analysis of ruminal bacterial community dynamics in lactating dairy cows during the feeding cycle. Anaerobe 16:94-100.

Wolin, M. J. 1960. A theoretical rumen fermentation balance. J. Dairy Sci. 40:1452-1459.

Yan, T., R. E. Agnew, and F. J. Gordon. 2002. The combined effects of animal species (sheep versus cattle) and level of feeding on digestible and metabolizable energy concentrations in grass silage based diets of cattle. Anim. Sci. 75:141-151.

Yan, T., R. E. Agnew, F. J. Gordon, and M. G. Porter. 2000. Prediction of methane energy output in dairy and beef cattle offered grass silage-based diets. Livest. Prod. Sci. 64:253-263.

Yan, T., C. S. Mayne, F. J. Gordon, M. G. Porter, R. E. Agnew, D. C. Patterson, C. P. Ferris, and D. J. Kilpatrick. 2010. Mitigation of enteric methane emissions through improving efficiency of energy utilization and productivity in lactating dairy cows. J. Dairy Sci. 93:2630-2638.

Yan, T., M. G. Porter, and C. S. Mayne. 2009. Prediction of methane emission from beef cattle using data measured in indirect open circuit respiration calorimeters. Animal 3:1455-1462. 